\title{
Escala de Motivos para Cesar/Reducir el Consumo de Alcohol: propiedades psicométricas
}

\author{
Reasons for Stop/Reduce Alcohol Consumption Scale: \\ Psychometric Properties
}

\author{
Karina Conde ${ }^{\mathrm{a}}$, Aldana Lichtenberger ${ }^{\mathrm{a}}$, Raquel Inés Peltzer ${ }^{\mathrm{b}}, \&$ Mariana Cremonte ${ }^{\mathrm{a}, \mathrm{b}}$ \\ ${ }^{a}$ Consejo Nacional de Investigaciones Científicas y Técnicas, Mar del Plata, Argentina \\ ${ }^{b}$ Universidad Nacional de Mar del Plata, Mar del Plata, Argentina
}

\begin{abstract}
Resumen: El objetivo de este estudio fue evaluar las propiedades psicométricas de una escala de motivos para cesar o reducir el consumo de alcohol en jóvenes universitarios, en cuanto a dimensionalidad, nivel del ítem, consistencia interna y validez concurrente. Participaron del estudio 377 estudiantes de psicología de la Universidad Nacional de Mar del Plata. Mediante un cuestionario anónimo y confidencial, se indagaron motivos para cesar o reducir el consumo de alcohol con una escala de 20 ítems, desarrollados a partir de respuestas cualitativas. Se incluyeron como criterios de validez la cantidad habitual de alcohol consumida y una regla de motivación al cambio. Se realizaron análisis factoriales exploratorios, confirmatorios, de confiabilidad y bivariados. Los análisis factoriales exploratorios y confirmatorios apuntaron a una estructura de tres dimensiones llamadas: rechazo del alcohol, salud y cambios vitales, y consecuencias severas del consumo de alcohol. Los coeficientes de confiabilidad fueron adecuados. Se encontraron relaciones entre las dos primeras dimensiones y la cantidad de alcohol consumida, y entre la motivación al cambio y la dimensión salud y cambios vitales. Se discute la diferencia entre motivos hipotéticos y reales para el cambio, y el rol de las consecuencias graves del consumo en grupos de mayor ingesta de alcohol.
\end{abstract}

Palabras clave: MCR-A, consumo de alcohol, universitarios, Argentina.
Abstract: The aim of this study was to evaluate the psychometric properties of a scale of motives to cease or reduce alcohol consumption in university students in terms of dimensionality, item level, internal consistency, and concurrent validity. The sample was constituted by 377 psychology students from the Universidad Nacional del Mar del Plata. Through an anonymous and confidential questionnaire, motives to cease or reduce alcohol consumption were enquired with a 20item scale developed from qualitative answers, and as validation criteria, the habitual alcohol quantity consumed and the motivation to change ruler. Exploratory and confirmatory factorial analyses, reliability and bivariated analysis were performed. The exploratory and confirmatory factorial analyses pointed to a three-dimensional structure labeled as alcohol rejection, health and vital changes, and severe consequences of alcohol consumption. Reliability coefficients were satisfactory. Relationships were found between the first two dimensions and the quantity of alcohol consumed, and between motivation to change and health and vital changes dimension. The difference between hypothetical and real motives for change and the role of severe consequences of alcohol consumption in groups of higher alcohol consumption are discussed.

Keywords: MCR-A, alcohol consumption, university, Argentina.

Este proyecto fue financiado, en parte, por el Consejo Nacional de Investigaciones Científicas y Técnicas y la Universidad Nacional de Mar del Plata.

Contacto: K. Conde. Complejo Universitario - Funes 3250 - Cuerpo V - Nivel III. Mar del Plata, Argentina. Correo electrónico:kconde@mdp.edu.ar

Cómo citar: Conde, K., Lichtenberger, A., Peltzer, R. I., \& Cremonte, M. (2016). Escala de Motivos para Cesar/Reducir el Consumo de Alcohol: propiedades psicométricas. Revista de Psicología, 25(2), 1-13.

http://dx.doi.org/10.5354/0719-0581.2016.42776 


\section{Introducción}

Las personas mantienen o cambian las conductas orientadas a cuidar o no de su salud por motivos que son parcialmente desconocidos. Una conducta cuyos daños en la salud propia y del entorno son ampliamente reconocidos es el consumo de alcohol (World Health Organization, 2010), el que, a pesar de grandes esfuerzos preventivos, sigue siendo un extenso problema para la salud pública en países menos desarrollados (Monteiro, 2013) y en grupos de alto riesgo como los jóvenes universitarios (Hingson, Heeren, Zakocs, Kopstein, \& Weschler, 2002).

En el estudio de cambios de hábitos de salud, se encuentra una línea de investigación que intenta responder por qué algunas personas interrumpen definitivamente (cesan) o reducen su consumo de alcohol (Amodeo \& Kurtz, 1998). Conocer cuáles son los motivos que conducen a este comportamiento puede ser tan importante para desarrollar medidas eficaces de prevención como para conocer las razones que llevan a una persona a comenzar a beber.

Sin embargo, los estudios que indagan sobre los motivos que llevarían a las personas a cesar o reducir su consumo de alcohol aún son escasos (Epler, Sher, \& Piasecki, 2009) y prácticamente inexistentes en el contexto latinoamericano (Conde, Peltzer, Lichtenberger, Tosi, \& Cremonte, 2016). Por otra parte, estas investigaciones utilizan metodologías muy diversas, dificultando la comparación. En algunos casos, se utilizan metodologías cualitativas con preguntas abiertas de difícil categorización (Cunningham, Blomqvist, Koski-Jännes, Cordingley, \& Callaghan, 2004); en otros, cuestionarios estructurados (Britton \& Bell, 2015; Epler et al., 2009; Oei, Swee- ton, Dingle, \& Chalmers, 1999), donde la cantidad y contenido de los ítems es muy heterogénea.

Adicionalmente, los motivos para cesar o reducir el consumo de alcohol varían de acuerdo a la muestra utilizada, el patrón de consumo (Epler et al., 2009) y la severidad de las consecuencias (Cunningham et al., 2004). De este modo, la mayor parte de los trabajos se ha centrado en consumidores patológicos, o consumidores excesivos, que han decidido cambiar su consumo de forma natural (Amodeo \& Kurtz, 1998; Carballo et al., 2014; Cunningham et al., 2004; Matzger, Kaskutas, \& Weisner, 2005). En estos grupos los cambios vitales importantes (Matzger et al., 2005), como haber obtenido una nueva responsabilidad, sentirse más maduro/a, querer mejorar la salud (Cunningham et al., 2004), haber recibido una advertencia por parte de su médico o familia, tener un despertar espiritual (Matzger et al., 2005) o haber experimentado un evento traumático (Blomqvist \& Cameron, 2002; Klingermann, 1991; Matzger et al., 2005; Tucker, Vuchinich, \& Gladsjo, 1994), se encontraron entre los motivos más frecuentes para realizar un cambio.

La sensación de "tocar fondo" pareció ser un motivo compartido también por adultos bebedores no patológicos, aunque quienes tenían problemas con el consumo refirieron más motivos (Matzger et al., 2005). Estos eventos que tienen un rol de transición en la vida de las personas son frecuentemente conceptualizados en inglés como maturing out, es decir, situaciones relacionadas con la maduración que ayudarían a la recuperación de los problemas por uso de alcohol, tanto por la reducción como por la abstinencia de consumir la sustancia (Dawson, Grant, Stinson, \& Chou, 2006). 
En menor medida, existen trabajos sobre la reducción o cese de consumo con poblaciones específicas, como adultos mayores (Britton \& Bell, 2015; Khan, Wilkinson, \& Keeling, 2006), jóvenes o estudiantes (Barnett, Goldstein, Murphy, Colby, \& Monti, 2006; Kuntsche, Knibbe, Gmel, \& Engels, 2005). Entre estudiantes universitarios, haber sufrido un evento traumático parecería asociarse con la decisión de disminuir el consumo (Barnett et al., 2006). En un estudio preliminar en el contexto latinoamericano, los jóvenes con mayor consumo y mayores consecuencias en el pasado argumentaron como motivos de disminución los cambios vitales, problemas interpersonales y espiritualidad. Mientras que en aquellos jóvenes con consumo leve, el motivo pareció estar principalmente asociado al rechazo hacia el sabor de las bebidas con alcohol (Conde et al., 2016).

Ante la gran variabilidad y la falta de instrumentos validados para medir la reducción o cese de consumo de alcohol en la juventud, en este trabajo se evaluaron las propiedades psicométricas de una escala construida con base en datos cualitativos, para estimar los motivos para cesar o reducir el consumo de alcohol en jóvenes universitarios, en términos de su dimensionalidad, funcionamiento a nivel del ítem, consistencia interna y validez concurrente, respecto de la cantidad de alcohol consumida y la motivación al cambio. Se espera, de este modo, contribuir al conocimiento sobre los cambios de comportamiento en el consumo de alcohol y al desarrollo de técnicas de prevención e intervención.

\section{Método}

\section{Participantes}

Se recurrió a una muestra por conveniencia de 377 estudiantes del primer y se- gundo año de la Licenciatura en Psicología de la Universidad Nacional de Mar del Plata (73\% mujeres; un estudiante se negó a contestar el cuestionario, este hubiese sido el participante número 378). Para determinar el tamaño muestral, se sobrestimó el mínimo necesario para realizar análisis factoriales (Osborne \& Costello, 2004), con una relación de 5:1 (cinco casos por ítem) para cada tipo de análisis (i.e., exploratorio y confirmatorio). La media de edad fue de 23 años $(D E=7,19)$. Se recolectaron datos de todos aquellos alumnos presentes en distintas clases entre septiembre y diciembre de 2015.

\section{Medidas}

Motivos para cesar o reducir el consumo de alcohol (MCR-A). Se evaluaron los MCR-A mediante una escala desarrollada a partir de respuestas cualitativas recabadas en un estudio previo. En una muestra de 1.708 estudiantes universitarios, se realizó una pregunta abierta a aquellas personas que habían cambiado su consumo de alcohol, indagando el motivo principal que los llevó a cesar o reducir su ingesta $(n=100)$. Con sus respuestas, se construyeron 16 categorías testeadas mediante acuerdo entre evaluadores (Kappa de Cohen $=, 84$ ) y cotejadas con literatura previa (Conde et al., 2016). La escala final estuvo constituida por 20 ítems, en los que se indagó qué nivel de importancia tenían para la persona cada uno de los motivos para cesar o reducir el consumo de alcohol. Las opciones de respuesta fueron ningu$n a=0$, poca $=1$ y mucha $=2$; y se evaluaron por separado los mismos 20 ítems en los motivos para cesar y para reducir el consumo de alcohol.

Consumo de alcohol. Se preguntó la cantidad habitual de alcohol consumida 
en unidades estándar, es decir, cualquier bebida alcohólica con 11 gr de alcohol puro. Se consideró como bebedor/a al momento de la investigación a aquellas personas que hubiesen consumido al menos una unidad estándar en los últimos 12 meses.

Motivación al cambio. Se estableció mediante una regla de motivación al cambio, en la cual los participantes debían colocar qué tan preparados estaban para cambiar su consumo de alcohol en un continuo de 10 valores, siendo $1=$ nada $\mathrm{y}$ $10=$ mucho . Este método es utilizado en las intervenciones breves para estimar la etapa de motivación al cambio de los pacientes (Bernstein, Bernstein, \& Levenson, 1997).

\section{Procedimiento}

Se convocó a los estudiantes en las aulas donde tomaban clases y la administración de la escala se realizó en ese mismo momento, con una duración aproximada de 30 minutos. La participación fue voluntaria y anónima, no se entregó ninguna clase de compensación y se obtuvo consentimiento informado. Al finalizar la administración de la escala, se entregaron hojas de información general del estudio, contactos de los investigadores responsables y de centros de asistencia a personas con trastorno por uso de alcohol. Durante todo el proceso, estuvieron presentes investigadores entrenados para responder eventuales preguntas sobre los ítems del cuestionario; estos no conocían previamente a los participantes. El cuestionario fue entregado a todos los alumnos presentes, sin embargo, se indicó que quienes no deseaban participar podían devolverlo en blanco. El proyecto contó con el aval del Comité de Ética del Instituto Nacional de Epidemiología.

\section{Análisis de datos}

La muestra fue dividida aleatoriamente en mitades, con el fin de realizar análisis exploratorios y confirmatorios. En una de estas mitades, se realizaron análisis factoriales exploratorios con los MCR-A por separado. Primero, se estimó la plausibilidad de realizar el análisis con las pruebas Kaiser-Meyer-Olkin y la prueba de esfericidad de Bartlett. El número de factores se valoró mediante la observación de los autovalores y por análisis paralelo (número de replicaciones $=100$, percentil de representación de simulaciones $=, 95$ ), reteniendo aquellos factores cuyo autovalor fuese superior al autovalor simulado. El método de extracción fue de máxima verosimilitud.

Tratándose de ítems ordinales, se realizaron análisis factoriales para una matriz de correlaciones policóricas; dada la correlación entre las dimensiones (mayor a 40 en algunos casos), se optó por el método de rotación de solución factorial promax. Los ítems finales se establecieron por la carga factorial (mayor a 40) y por la correlación puntual biserial corregida en la dimensión a la cual pertenecían. Una vez establecidos los ítems, se repitió el análisis factorial exploratorio para corroborar la estructura con el nuevo conjunto de ítems (los resultados finales de este proceso se encuentran en la tabla 1), y se prosiguió con el análisis de consistencia interna (alfa de Cronbach) para cada una de las dimensiones. 
Tabla 1

Análisis a nivel del ítem de la escala MCR-A

\begin{tabular}{|c|c|c|c|c|}
\hline \multirow[b]{2}{*}{ Ítems } & \multicolumn{2}{|c|}{ Motivos para cesar } & \multicolumn{2}{|c|}{ Motivos para reducir } \\
\hline & $\beta$ & $\begin{array}{l}\text { Correlación } \\
\text { biserial puntual } \\
\text { corregida }\end{array}$ & $\beta$ & $\begin{array}{c}\text { Correlación } \\
\text { biserial puntual } \\
\text { corregida }\end{array}$ \\
\hline Rechazo del alcohol & & & & \\
\hline 1. Desagrado &, 75 &, 53 &, 75 &, 53 \\
\hline 2. Falta de interés & ,83 & ,61 & ,99 & ,71 \\
\hline 3. Creencias religiosas o espiritualidad &, 50 & ,37 &, 56 & ,39 \\
\hline Salud y cambios vitales & & & & \\
\hline 4. Medicación o enfermedad & ,38 & ,33 & - & - \\
\hline $\begin{array}{l}\text { 5. Cambios personales, de rutina o nuevos } \\
\text { roles }\end{array}$ &, 58 & ,48 & ,40 & ,40 \\
\hline 6. Potenciales daños o efectos en la salud & ,78 &, 52 &, 59 & ,45 \\
\hline 7. Practicar algún deporte o ejercicio físico & ,96 & 62 & 1,02 &, 54 \\
\hline 8. Evitar el aumento de peso o dieta & ,63 & ,49 & ,49 & ,41 \\
\hline 9. Evitar malestar corporal &, 78 & ,62 & ,63 &, 53 \\
\hline $\begin{array}{l}\text { Consecuencias severas del consumo de alcohol } \\
\text { 10. Evitar malestar psíquico }\end{array}$ & ,48 &, 57 &, 57 &, 54 \\
\hline 11. Relaciones familiares o de pareja &, 75 & ,62 &, 73 &, 65 \\
\hline $\begin{array}{l}\text { 12. Actividades diarias como el estudio o } \\
\text { trabajo }\end{array}$ & ,63 &, 59 & ,72 & ,62 \\
\hline $\begin{array}{l}\text { 13. Notar diferencias positivas en sí mismo } \\
\text { cuando no está bajo los efectos del alcohol }\end{array}$ & ,60 & ,61 & ,73 & ,60 \\
\hline $\begin{array}{l}\text { 14. Notar diferencias en sí mismo cuando } \\
\text { no está bajo los efectos del alcohol y } \\
\text { otras personas sí }\end{array}$ &, 52 & ,46 & ,61 &, 56 \\
\hline 15. Sensación de perder la cordura & ,85 & ,65 & ,87 & ,63 \\
\hline 16. Evitar padecer dependencia del alcohol & 1,00 & ,67 & ,93 & ,68 \\
\hline 17. No concurrir a eventos sociales o fiestas &, 55 & ,43 & , 45 & ,36 \\
\hline 18. Evitar multas & 65 & ,46 & ,80 &, 51 \\
\hline $\begin{array}{l}\text { 19. Familiar directo de personas con de- } \\
\text { pendencia }\end{array}$ & ,62 & ,41 & ,58 & ,46 \\
\hline
\end{tabular}

Nota: Las correlaciones fueron calculadas con base en la dimensión correspondiente; $\beta=$ Carga factorial; - = Ítem eliminado de la escala.

Por otra parte, en la segunda mitad de la muestra se efectuaron análisis factoriales confirmatorios de los MCR-A por separado. Nuevamente, por el carácter ordinal de los ítems, las estimaciones se realizaron con el estimador mínimos cuadrados ponderados diagonalmente (diagonally weighted least squares). Para cada ítem, 
se tuvo en cuenta los coeficientes estandarizados. El ajuste de las dimensiones se estableció con el índice de ajuste comparativo (CFI, por su nombre en inglés Comparative Fit Index), el índice Tucker-Lewis (TLI, por su nombre en inglés Tucker-Lewis Index), el error cuadrático medio de aproximación (RMSEA, por su nombre en inglés Root Mean Square Error of Approximation) y la raíz estandarizada del error medio cuadrático (SRMR, por su nombre en inglés Standardized Root Mean Square Residual). Por último, se realizaron análisis de correlación (método Spearman, para datos no paramétricos) entre las escalas y dimensiones, y la cantidad de alcohol consumida y la motivación al cambio en aquellos participantes que eran bebedores actuales.

Para los análisis se utilizaron los paquetes estadísticos psych, funciones fa.parallel.poly y fa.poly (Revelle, 2012), y lavaan, función cfa (Rosseel, 2012), del software R 3.2.3, además del programa ICalfa (Merino, 2015). Se realizó imputación múltiple sobre los datos perdidos con el paquete MICE (por su nombre en inglés Multivariate Imputation by Chained Equations) del software R 3.2.3 (Buuren \& Oudshoorn, 2000).

\section{Resultados}

\section{Análisis factorial exploratorio}

La medida de adecuación KMO y la prueba de esfericidad de Bartlett fueron satisfactorias para los motivos para cesar $(\mathrm{KMO}=0,84$, prueba de esfericidad de Bartlett $=, 01)$ y para reducir $(\mathrm{KMO}=0,83$, prueba de esfericidad de Bartlett $=, 01)$ el consumo de alcohol. La valoración de los autovalores mediante el análisis paralelo, indicó que la mejor solución era la de tres factores, tanto para cesar como para reducir el consumo de alcohol (figura 1).

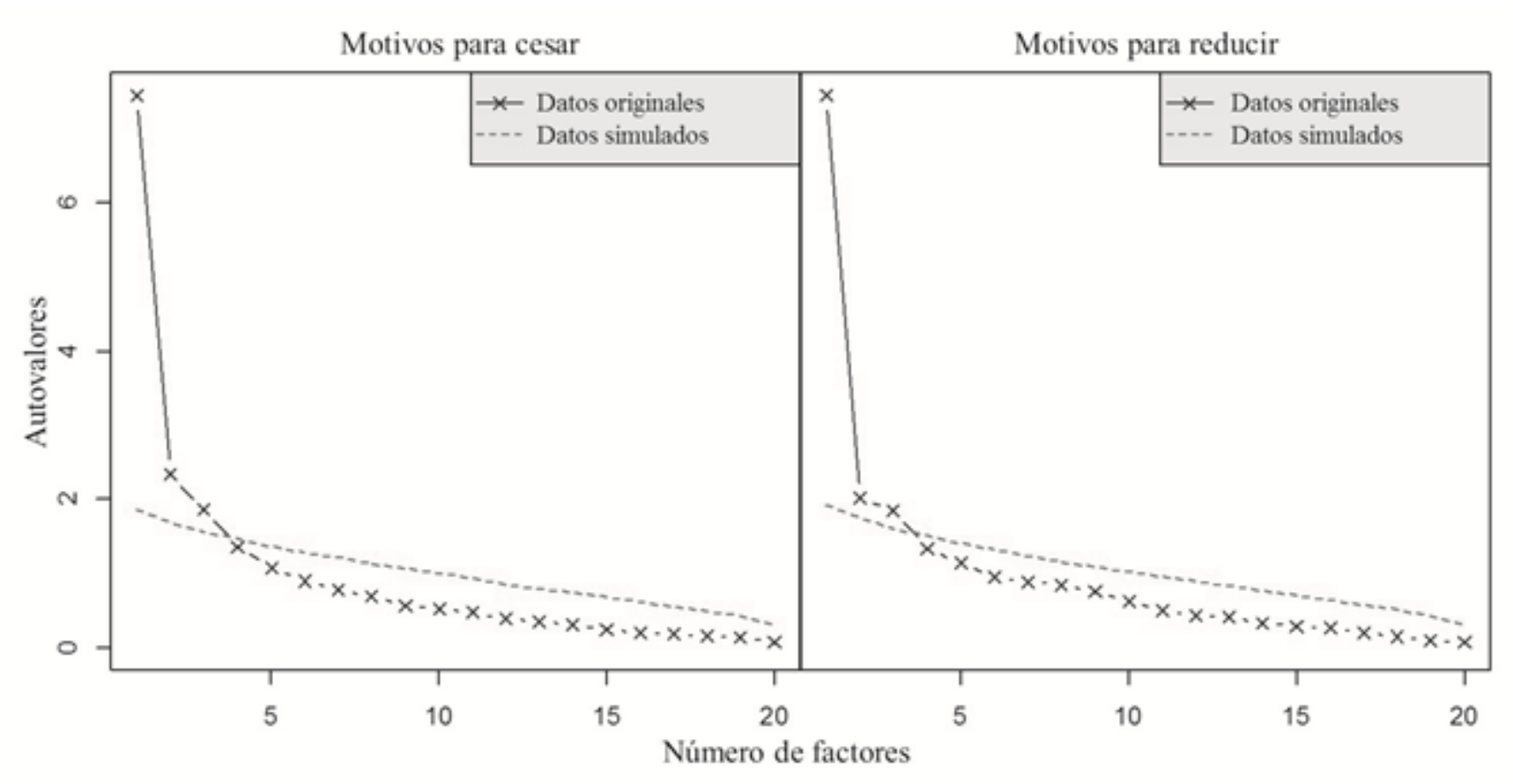

Figura 1. Autovalores y análisis paralelo de la escala MCR-A. 
Estos tres factores explicaron un 52\% y un $51 \%$ de la varianza respectivamente y, por las características de los ítems que agruparon (los mismos tanto para cesar como para reducir el consumo de alcohol, ver tabla 1), las dimensiones fueron llamadas: rechazo del alcohol (cesar, $M=$ 2,64 [IC 95\% 2,46; 2,82], reducir, $M=$ 2,75 [IC 95\% 2,57; 2,93]), salud y cambios vitales (cesar, $M=8,23$ [IC 95\% 7,$92 ; 8,51$ ], reducir, $M=6,95$ [IC 95\% $6,68 ; 7,21])$, y consecuencias severas del consumo de alcohol (cesar, $M=11,64$ [IC $95 \% 11,01 ; 12,19]$, reducir, $M=12,15$ [IC 95\% 11,59; 12,69]).

Las cargas factoriales y la correlación puntual biserial corregida fueron adecuadas para todos los ítems; con excepción del ítem por el precio de las bebidas con alcohol, en los motivos para cesar y reducir el consumo de alcohol; y del ítem por ingerir alguna medicación o padecer una enfermedad que me impidiera consumir alcohol, en los motivos para reducir. Estos ítems fueron descartados de la escala final según el caso. También se detectó la presencia de un caso Heywood (ítems con comunalidades iguales a 1) en cada escala (tabla 1).

\section{Confiabilidad}

Las dimensiones mostraron consistencia interna satisfactoria, menor en la dimensión rechazo del alcohol (cesar, 3 ítems, $\alpha=, 66$, [IC 95\%,59; ,71]; reducir, 3 ítems, $\alpha=, 67$, [IC 95\%,61; ,72]), intermedia la dimensión salud y cambios vitales (cesar, 6 ítems, $\alpha=$ ,74, [IC 95\%,67; ,79]; reducir, 5 ítems, $\alpha=$ ,71, [IC 95\%,64; ,77]), y mayor en la dimensión consecuencias adversas del consumo de alcohol (cesar, 10 ítems, $\alpha=, 86$, [IC 95\% ,83; ,89]; reducir, 10 ítems, $\alpha=$ ,86, [IC 95\%,83; ,89]).

\section{Análisis factorial confirmatorio}

El análisis factorial confirmatorio en la segunda mitad de la muestra presentó índices satisfactorios en la escala de motivos para cesar el consumo de alcohol, $\chi^{2}$ $=1146,42, g l=14, p<, 05, \mathrm{CFI}=0,92$, $\mathrm{TLI}=0,91, \mathrm{RMSEA}=0,07, \mathrm{SRMR}=0,07$, y óptimos en la escala de motivos para reducirlo, $\chi^{2}=1118,25, g l=153, p<, 05$, $\mathrm{CFI}=0,96, \mathrm{TLI}=0,95, \mathrm{RMSEA}=0,04$, SRMR $=0,05$. Los coeficientes estandarizados de cada ítem y de las dimensiones respecto de la escala total, se encuentran en la figura 2.

\section{Validez concurrente}

En los bebedores actuales $(n=308)$ se encontraron correlaciones leves entre los motivos para cesar $(r=-, 12$, [IC $95 \%-, 02 ;-, 24], p<, 05)$ y reducir $(r=-$ ,14, [IC 95\% -,04; -,25], $p<, 05)$, y la cantidad de alcohol consumida. En el análisis según las dimensiones, se encontró que rechazo del alcohol (cesar, $r$ $=-, 14$, [IC 95\% -,02; -,24], $p<, 05$; reducir, $r=-, 18$, [IC 95\% -,07; -,28], $p<$ ,01) y salud y cambios vitales (cesar, $r=$ -,18, [IC 95\% -,07; -,28], p < ,01; reducir, $r=-, 18$, [IC 95\% -,7; -,29], $p<, 05$ ) se relacionaron con la cantidad de alcohol consumida, pero no así la dimensión consecuencias severas del consumo de alcohol.

Respecto de la motivación al cambio, nuevamente, tanto en los motivos para cesar como en los motivos para reducir el consumo de alcohol, solo se relacionó levemente con la dimensión salud y cambios vitales (cesar, $r=, 11$, [IC 95\% 0; ,23], $p<, 05$; reducir, $r=, 12$, [IC 95\% $, 01 ;, 23], p<, 05)$. 


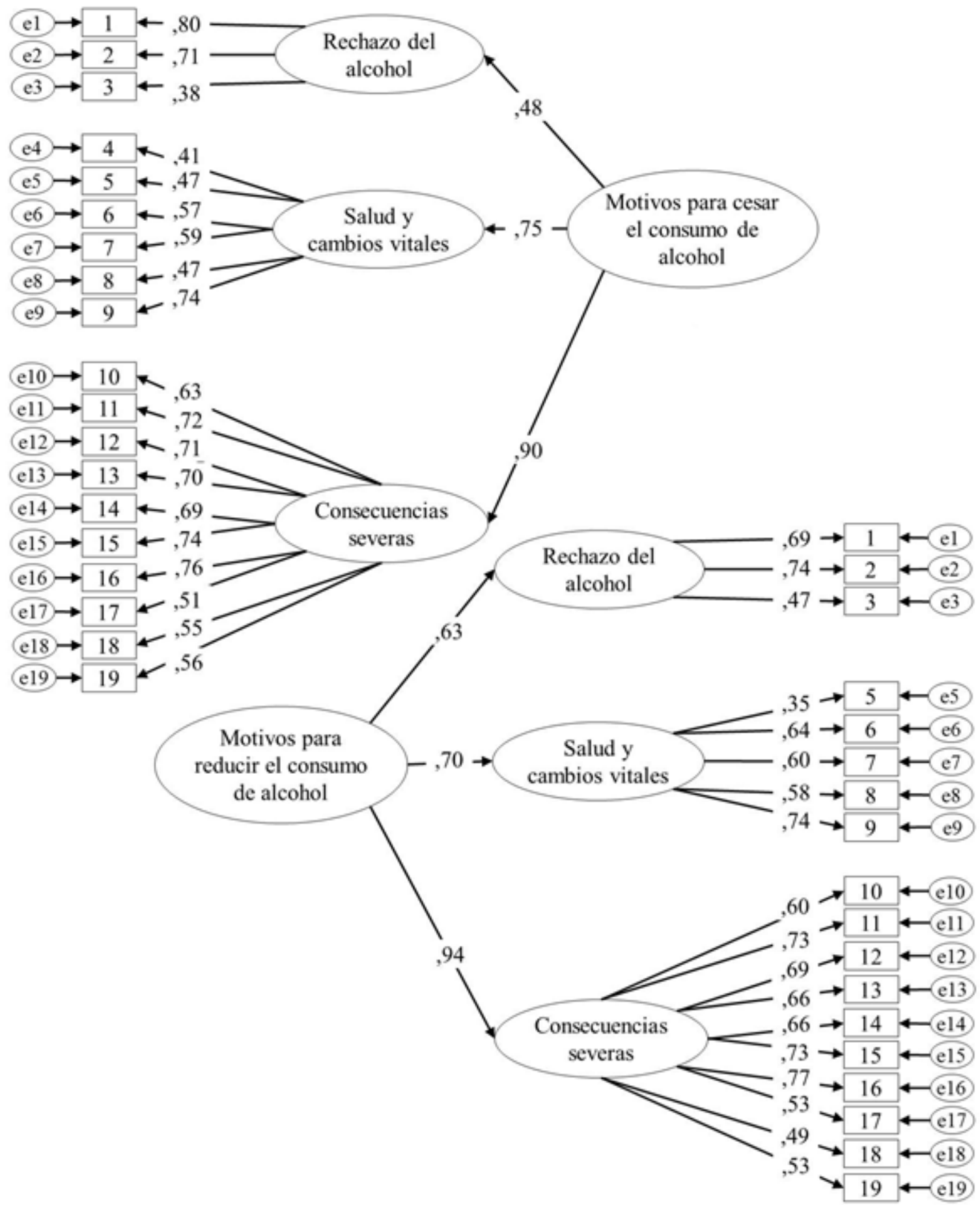

Figura 2. Coeficientes estandarizados para el análisis factorial confirmatorio de la escala MCR-A.

\section{Discusión}

A nivel internacional se ha establecido la importancia de desarrollar medidas adecuadas sobre los motivos para cambiar el consumo de alcohol en la juventud (Anderson, Grunwald, Bekman, Brown, \& Grant, 2011); pues los avances en esta temática son escasos, particularmente en Latinoamérica. De allí se desprendió el 
objetivo de este trabajo, con el fin de evaluar las propiedades psicométricas de una escala construida especialmente para estimar los motivos para cesar o reducir el consumo de alcohol en jóvenes universitarios.

Los análisis realizados sobre los 20 ítems iniciales arrojaron una estructura de tres dimensiones: rechazo del alcohol, salud y cambios vitales, y consecuencias severas del consumo de alcohol. La primera dimensión estuvo conformada por razones que llevan al rechazo de la sustancia, ya sea por disgusto, indiferencia o porque sus creencias religiosas o espirituales se contraponen al consumo. La segunda dimensión incluyó razones relacionadas con el cuidado de la salud física, evitar perjuicios, empeorar enfermedades y con cambios vitales personales, de rutina o de roles. La tercera incluyó motivaciones relacionadas con las consecuencias severas del consumo de alcohol, padecimientos en la salud mental (como la dependencia), la reflexión sobre sí mismos/as y los cambios cuando se consume alcohol, problemas interpersonales y multas. También incluyó la falta de participación en eventos sociales, lo que puede ser parcialmente explicado en que esta categoría incorporó razones con un importante componente emocional y/o cognitivo, y la asistencia a eventos sociales puede implicar una presión importante de los pares para consumir alcohol (Balsa, Homer, French, \& Norton, 2011; Jamison \& Myers, 2008; Sanhueza, Delva, Bares, \& Grogan-Kaylor, 2013).

Aunque esta solución factorial detectó un caso Heywood en cada escala, estos son comunes en los estudios factoriales con correlaciones policóricas (Freiberg Hoffmann, Stover, de la Iglesia, \& Fernández Liporace, 2013); asimismo, las tres dimensiones tendrían un sentido teórico, y las pruebas de consistencia interna y el análisis factorial confirmatorio fueron satisfactorios.

La cantidad inicial de ítems se redujo a 19 para los motivos para cesar y 18 en los motivos para reducir el consumo de alcohol. El precio de las bebidas alcohólicas no pareció formar parte de las dimensiones para cesar o reducir el consumo de alcohol, aunque la evidencia empírica sugiere que aumentar los precios es una medida importante para reducir el consumo de alcohol y los daños ocasionados por el mismo (Babor, 2010). En este punto podría discutirse que la escala, si bien está construida con base en motivos reales, para muchos evalúa motivos hipotéticos para cesar o reducir el consumo de alcohol, que podrían ser distintos a los que llevan a reducir o cesar el consumo en la práctica. Por otra parte, también es posible que los motivos de aquellos que consumen alcohol sean distintos a los de quienes decidieron modificar el consumo. Futuras investigaciones podrían ocuparse de dilucidar esta cuestión.

Por otro lado, las razones médicas (consumir un medicamento o padecer una enfermedad) no formaron parte de los motivos para reducir el consumo de alcohol, por lo que podría pensarse que, en términos de dimensionalidad, juega un rol en la estructura de los motivos para cesar el consumo de alcohol, pero no en reducirlos.

El análisis de validez concurrente indicó que la cantidad de alcohol consumida se relaciona negativamente con las dimensiones rechazo del alcohol y salud y cambios vitales, pero no con consecuencias graves del consumo. Esto podría deberse a que las personas con mayor consumo no consideran relevantes las primeras dos dimensiones, pero la tercera es igualmente relevante, sin importar el nivel de consumo. 
Por lo tanto, profundizar este resultado puede ser valioso si se entiende que las dimensiones rechazo del alcohol y salud y cambios vitales puede derivar en estrategias destinadas a grupos de bajo consumo; y, por otra parte, la dimensión consecuencias graves del consumo pueden derivar en estrategias para las personas con mayor consumo. De hecho, la última dimensión, consecuencias graves del consumo, contiene una gran cantidad de motivos similares a los reportados en estudios realizados a personas con trastorno por uso de alcohol y consumidores excesivos. Sin embargo, algunos motivos que se observaron en estos grupos en otros contextos, como las razones espirituales o los cambios vitales, no se incluyeron en esta dimensión (Amodeo \& Kurtz, 1998; Cunningham et al., 2004; Matzger et al., 2005).

En este sentido, podría darse el caso de que los motivos para cesar o reducir el consumo actuaran de manera diferente en distintos contextos. Por ejemplo, en Argentina el consumo de alcohol es ampliamente aceptado, por lo que no se esperaría que las personas dejen de consumir, a pesar de sus creencias religiosas o por cambios de roles; en cambio, el estigma y el temor asociado a padecer consecuencias adversas del consumo, como un trastorno mental, podría ser una invariante cultural.

Finalmente, otro resultado interesante es que la motivación al cambio se asoció positivamente solo con respecto a la dimensión salud y cambios vitales. Esto podría explicarse, teniendo en cuenta que los ítems que componen la dimensión incluyen cuestiones realizadas con el cuidado activo de la salud; por tal razón los puntajes más altos en esta dimensión podrían indicar personas más motivadas a cuidar su salud y, por lo tanto, a cambiar factores perjudiciales para esta.

Además de las limitaciones propias de la imposibilidad de generalización de lo hallado, que significan que este estudio es únicamente aplicable a poblaciones de similares características, el uso de correlaciones policóricas es una opción de análisis que podría complementarse en nuevos estudios con la estimación de umbrales de transición.

Por otro lado, los motivos para cesar o reducir el consumo podrían ser causantes de la motivación al cambio, es decir, tener un efecto sobre la motivación al cambio, y no el resultado de un constructo subyacente. $\mathrm{O}$ incluso se puede hipotetizar que algunos de estos motivos, sobre todo aquellos que resultan de eventos vitales, tienen un efecto de retroalimentación con el constructo de motivación a cesar o reducir, similar al hallado en otros estudios (Arterberry, Chen, Vergés, Bollen, \& Martens, 2015). Tales relaciones y otras más complejas podrían explorarse en análisis posteriores y en versiones futuras de la escala. Debe considerarse, por ejemplo, que algunas dimensiones contienen tan solo tres ítems, y una versión más extensa del instrumento podría beneficiar la comprensión de la problemática.

Otra limitación importante a este estudio es la falta de medidas recomendables para establecer la validez concurrente, lo cual podría reflejarse en el hecho de que la dimensión consecuencias severas no presentó correlaciones con ninguna de las medidas utilizadas (cantidad y motivación al cambio).

Más allá de las limitaciones, esta investigación representa una contribución relevante a un campo poco explorado dentro 
del consumo de sustancias psicoactivas en los jóvenes, especialmente el consumo de alcohol. Además, aporta un instrumento construido y evaluado en el contexto local para explorar las relaciones entre los motivos para cesar o reducir el consumo de alcohol y distintas variables en una población de riesgo. Un ejemplo son las expec- tativas hacia el consumo de alcohol, para cuya evaluación se han confeccionado y validado instrumentos específicos con población juvenil argentina (Pilatti, Godoy, \& Brussino, 2012). Asimismo, podrían considerarse otras poblaciones en las cuales explorar los motivos para abandonar o reducir el consumo de alcohol.

\section{Referencias}

Amodeo, M. \& Kurtz, N. R. (1998). Coping methods and reasons for not drinking. Substance Use \& Misuse, 33(8), 1591-1610. http://dx.doi.org/10.3109/10826089809058946

Anderson, K. G., Grunwald, I., Bekman, N., Brown, S. A., \& Grant, A. (2011). To drink or not to drink: Motives and expectancies for use and nonuse in adolescence. Addictive Behaviors, 36(10), 972-979.

http://dx.doi.org/10.1016/j.addbeh.2011.05.009

Arterberry, B. J., Chen, T. -H., Vergés, A., Bollen, K. A., \& Martens, M. P. (2015). How should alcohol problems be conceptualized? Causal indicators within the Rutgers Alcohol Problem Index. Evaluation \& the Health Professions, 39(3), 356-378. http://dx.doi.org/ 10.1177/0163278715616440

Babor, T. (2010). Alcohol: No ordinary commodity - Research and public policy. Oxford, United Kingdom: Oxford University Press.

Balsa, A. I., Homer, J. F., French, M. T., \& Norton, E. C. (2011). Alcohol use and popularity: Social payoffs from conforming to peers' behavior. Journal of Research on Adolescence, 21(3), 559-568. http://dx.doi.org/10.1111/j.1532-7795.2010.00704.x

Barnett, N. P., Goldstein, A. L., Murphy, J. G., Colby, S. M., \& Monti, P. M. (2006). "I'll never drink like that again": Characteristics of alcohol-related incidents and predictors of motivation to change in college students. Journal of Studies on Alcohol, 67(5), 754-763. http://dx.doi.org/10.15288/jsa.2006.67.754

Bernstein, E., Bernstein, J., \& Levenson, S. (1997). Project ASSERT: an ED-based intervention to increase access to primary care, preventive services, and the substance abuse treatment system. Annals of Emergency Medicine, 30(2), 181-189. http://dx.doi.org/10.1016/S0196-0644(97)70140-9

Blomqvist, J. \& Cameron, D. (2002). Editorial: Moving away from addiction: Forces, processes and context. Addiction Research and Theory, 10(2), 115-118. http://dx.doi.org/10.1080/16066350290017211

Britton, A. \& Bell, S. (2015). Reasons why people change their alcohol consumption in later life: Findings from the Whitehall II cohort study. PloS One, 10(3), 1-8. http://dx.doi.org/10.1371/journal.pone.0119421 
Buuren, S. V. \& Oudshoorn, C. G. M. (2000). Multivariate imputation by chained equations: MICE V1. 0 user's manual (No. PG/VGZ/00.38). Leiden, Netherlands: TNO Prevention and Health

Carballo, J. L., Sobell, L. C., Dum, M., Sobell, M. B., Fernández-Hermida, J. R., SecadesVilla, R., \& García-Rodríguez, O. (2014). Self-change among Spanish speakers with alcohol and drug use disorders in Spain and the United States. Addictive Behaviors, 39(1), 225-230.

http://dx.doi.org/10.1016/j.addbeh.2013.10.013

Cunningham, J. A., Blomqvist, J., Koski-Jännes, A., Cordingley, J., \& Callaghan, R. (2004). Characteristics of former heavy drinkers: Results from a natural history of drinking general population survey. Contemporary Drug Problems, 31(2), 357-369. http://dx.doi.org/10.1177/009145090403100208

Conde, K., Peltzer, R.I., Lichtenberger, A., Tosi, L., \& Cremonte, M. (2016). Razones para cambiar el consumo de alcohol en estudiantes universitarios. Manuscrito no publicado.

Dawson, D. A., Grant, B. F., Stinson, F. S., \& Chou, P. S. (2006). Maturing out of alcohol dependence: The impact of transitional life events. Journal of Studies on Alcohol, 67(2), 195-203.

http://dx.doi.org/10.15288/jsa.2006.67.195

Epler, A. J., Sher, K. J., \& Piasecki, T. M. (2009). Reasons for abstaining or limiting drinking: A developmental perspective. Psychology of Addictive Behaviors, 23(3), 428-442. http://dx.doi.org/10.1037/a0015879

Freiberg Hoffmann, A., Stover, J. B., de la Iglesia, G., \& Fernández Liporace, M. (2013). Correlaciones policóricas y tetracóricas en estudios factoriales exploratorios y confirmatorios. Ciencias Psicológicas, 7(2), 151-164. Recuperado de https://is.gd/jLBfjs

Hingson, R. W., Heeren, T., Zakocs, R. C., Kopstein, A., \& Wechsler, H. (2002). Magnitude of alcohol-related mortality and morbidity among US college students ages 18-24: Changes from 1998 to 2001. Journal of Studies on Alcohol, 63(2), 136-144. http://dx.doi.org/10.15288/jsa.2002.63.136

Jamison, J. \& Myers, L. B. (2008). Peer-group and price influence students drinking along with planned behaviour. Alcohol and Alcoholism, 43(4), 492-497. http://dx.doi.org/10.1093/alcalc/agn033

Khan, N., Wilkinson, T. J., \& Keeling, S. (2006). Reasons for changing alcohol use among older people in New Zealand. Australasian Journal on Ageing, 25(2), 97-100. http://dx.doi.org/10.1111/j.1741-6612.2006.00159.x

Klingermann, H. K. H. (1991). The motivation for change from problem alcohol and heroin use. British Journal of Addiction, 86(6), 727-744. http://dx.doi.org/10.1111/j.1360-0443.1991.tb03099.x

Kuntsche, E., Knibbe, R., Gmel, G., \& Engels, R. (2005). Why do young people drink? A review of drinking motives. Clinical Psychology Review, 25(7), 841-861. http://dx.doi.org/10.1016/j.cpr.2005.06.002 
Matzger, H., Kaskutas, L. A., \& Weisner, C. (2005). Reasons for drinking less and their relationship to sustained remission from problem drinking. Addiction, 100(11), 16371646.

http://dx.doi.org/10.1111/j.1360-0443.2005.01203.x

Merino, C. (2015). ICalfa: módulo Excel para estimar intervalos de confianza para alfa de Cronbach. [Software de Computadora]. Lima, Perú: Universidad de San Martín de Porres.

Monteiro, M. G. (2013). Alcohol y salud pública en América Latina: ¿cómo impedir un desastre sanitario? Adicciones, 25(2), 99-105.

http://dx.doi.org/10.20882/adicciones.56

Oei, T. P. S., Sweeton, J., Dingle, G. A., \& Chalmers, K. A. (1999). Psychometric properties of a quitting time for alcohol questionnaire: Factor structure, reliability, and validity. Addictive Behaviors, 24(3), 383-398.

http://dx.doi.org/10.1016/S0306-4603(98)00077-X

Osborne, J. W. \& Costello, A. B. (2004). Sample size and subject to item ratio in principal components analysis. Practical Assessment, Research \& Evaluation, 9(11). Recuperado de http://PAREonline.net/getvn.asp?v=9\&n=11

Pilatti, A., Godoy, J. C., \& Brussino, S. A. (2012). Adaptación de instrumentos entre culturas: ejemplos de procedimientos seguidos para medir las expectativas hacia el alcohol en el ámbito argentino. Trastornos Adictivos, 14(2), 58-64.

http://dx.doi.org/10.1016/S1575-0973(12)70045-4

Revelle, W. (2012). psych: Procedures for personality and psychological research. Evanston, Ilinois: Northwestern University. Recuperado de

https://CRAN.R-project.org/package=psych

Rosseel, Y. (2012). lavaan: An R package for structural equation modeling. Journal of Statistical Software, 48(2), 1-36.

http://dx.doi.org/10.18637/jss.v048.i02

Sanhueza, G. E., Delva, J., Bares, C., \& Grogan-Kaylor, A. (2013). Alcohol consumption among Chilean adolescents: Examining individual, peer, parenting and environmental factors. The International Journal of Alcohol and Drug Research, 2(1), 89-97. http://dx.doi.org/10.7895/ijadr.v2i1.71

Tucker, J. A., Vuchinich, R. E., \& Gladsjo, J. A. (1994). Environmental events surrounding natural recovery from alcohol-related problems. Journal of Studies on Alcohol, 55(4), $401-411$.

http://dx.doi.org/10.15288/jsa.1994.55.401

World Health Organization. (2010). Global strategy to reduce the harmful use of alcohol. Geneva, Switzerland: WHO Press. 